\title{
Genetic Separation of Chalkiness in the Hybrid Rice
}

\section{Lu GAN ${ }^{1}$, Xiao-shu DENG ${ }^{1}$, Yan LIU ${ }^{1}$, Jiao CHEN $^{1}$, Ru-yu TANG ${ }^{1}$, Li-xia LEI ${ }^{1}$, Jiang-hong TANG ${ }^{1}$, Jian-ni ZHANG $^{1}$ and Zheng-wu $\mathrm{ZHAO}{ }^{1,2, \mathrm{a},{ }^{*}}$}

${ }^{1}$ College of life sciences, Chongqing Normal University, Chongqing, China

${ }^{2}$ Chongqing Engineering Research Center of Specialty Crop Resources, Chongqing, China

azhaozhengwu513@sina.com

${ }^{*}$ Corresponding author

Keywords: Rice, Percentage of grians with chalkiness, Genetic segregation

\begin{abstract}
Percentage of grains with chalkiness (PGWC) controlled by multi-genes is one of the main appearance quality of rice. The selection among the offspring using genetic separation directly influences quality improvements of hybrid rice. The present study focused on the segregation of PGWC, using Huanghuazhan as the female parent and CS197 as the male parent to construct hybrid rice $F_{2}$ population. Molecular markers were used for genotype analysis among the extremely low and high PGWC individuals from the $\mathrm{F}_{2}$ population. The results revealed that the genotypes of 10 extremely low PGWC individual plants were $80.00 \%$ and $3.33 \%$ identical to that of Huanghuazhan and CS197, respectively. Genotype and phenotype of the most 10 individual plants were similar to Huanghuazhan. On the contrary, the genotypes of 10 extremely high PGWC individual plants were $37.78 \%$ and $32.22 \%$ identical to that of Huanghuazhan and CS197, respectively. Genotype and phenotype of the most of 10 individual plants were the similar to CS197. The extremely low and high PGWC individuals had high coincidence with the corresponding $\mathrm{F}_{2: 3}$ family lines in phenotype and genotype. This is the first report on the genetic separation of PGWC in Huanghuazhan.
\end{abstract}

\section{Introduction}

Over the past half century, rice production has made a second major leap, so that rice basically meets people's demand for food [1]. However, rice breeders ignored the improvement of rice quality, which caused the poor quality of cultivated varieties. Imported rice with good quality has impacted on China's rice industry. Therefore, improving the international competitiveness of China's rice quality is becoming the main goal of Chinese scientific research workers. High PGWC is one of the most important factors of poor rice quality [2-3]. In the practice of hybrid rice breeding, the genetic separation of $F_{2}$ generation of hybrids was the largest. The selection of strain was difficult, especially the quantitative traits. The PGWC is a quantitative trait controlled by multiple genes. Therefore, it is important to study the genetic separation of PGWC for the breeding of new high quality hybrid rice varieties and the creation of materials.

Rice chalkiness is resulted from insufficient seed filling influencing the light transmission [4]. Studies have shown that PGWC is a quantitative trait controlled by multiple genes. Kuo et al. [4] revealed that chalkiness was significantly affected by additive and dominance effects, and the additive effect was more important than the 
dominant effect, based on a $6 \times 6$ complete double-row hybridization design. Huang et al. [5] studied the compounding ability of indica rice. The results showed that the chalkiness and grain strength were affected by the general combining effect. In another word, the effect of gene additive effect was small, whereas the effect of special combining effect was greater.

The present study focused on the coincidence mechanism of individual phenotype and genotype of hybrid $\mathrm{F}_{2}$ isolates, which were constructed by the high quality rice cultivar Huanghuazhan as the female parent and CS197 as the male parent, using phenotypic identification, molecular markers, chalky starch morphological observation by SEM. The aim is to lay the foundation for the breeding of high quality varieties.

\section{Materials and Methods}

\section{Plant Materials and Field Experiment}

Huanghuazhan with low PGWC from the Rice Research Institute of Guangdong Academy of Agricultural Sciences was used as the female parent. CS197 with high PGWC from Chongqing Normal University was used as the male parent. In winter of 2013, the group of hybrid combinations was constructed in Hainan. In the spring of 2014-2016, the $F_{1}, F_{2}$ and $F_{2: 3}$ generations were planted at the Bishan Mountain Experimental Base of Chongqing Normal University. $F_{2}$ hybrid population with 120 individuals was constructed from Huanghuazhan and CS197.

\section{Trait Measurement of PGWC}

The measurements of PGWC of rice were followed by the "High-quality Rice" of People's Republic of China National Standard (GB/T17981-1999) determination of PGWC. One hundred samples $(\mathrm{N})$ were randomly selected from the whole milled rice samples and then placed on the chalky observation equipment to count those chalky rice grains and calculated PGWC. After three parallel, the average value was used as the trait value.

\section{DNA Extraction and SSR Labeling}

DNAs were extracted using the CTAB [6] method and agarose gel elec-trophoresis.

\section{Results}

\section{Chalky Grain Fraction Molecular Genetic Separation}

Nine of the 46 primer pairs with polymorphism between the parents (i.e. Huang Huazhan and CS197) were finally screened. The markers were consistent with the genotype of Huang Huazhan (white bars), and of CS197 (black bars) (Fig 1A). The proportion of the heterozygous genotype (diagonal bars, Fig 1A) in 10 grains with low PGWC was $80.00 \%, 3.33 \%$ and $16.67 \%$, respectively. The proportion the heterozygous genotype (diagonal bars, Fig 1A) in 10 grains with high PGWC was $37.78 \%, 32.22 \%$ and $30.00 \%$, respectively. The chalky grain rate of the chalky chrysanthemum was $5.66-11.00 \%$, with an average of $9.00 \%$, which was consistent with the rate of chrysanthemum grain rate of $10.32 \%$. The chalky grain rate of chalky chrysanthemum was $75.00-82.67 \%$, with an average of $78.90 \%$, which was close to the parental CS197 chalky grain rate of $81.67 \%$. 
The $\mathrm{F}_{2: 3}$ families of $\mathrm{F}_{2}$ population were identified by molecular marker and phenotypic identification. The results showed that $\mathrm{F}_{2: 3}$ families with low chalky grain rate of $\mathrm{F}_{2}$ population were still expressed as low chalky grain fraction of 3.67-27.33\%, with an average of $12.80 \%$. The chalkiness rate of the majority of families was slightly increased, and the number of families was slightly decreased. The results of the two generations were similar. In particular, the 9 markers detected the markers consistent with the genotype of Huanghuazhan (Fig $1 \mathrm{~A}, \mathrm{~B}) . \mathrm{F}_{2}$ population of 10 high chalky grain rate extreme single plant corresponding $\mathrm{F}_{2: 3}$ family of phenotype and genotype similarity is higher. The markers consistent with the genotype of the two extremes were similar to those in $F_{2}$ and $F_{2: 3}$ families. RM1297, RM1068, RM1378, RM234 ratio is greater than 3, more suitable for PGWC of molecular marker assisted selection.
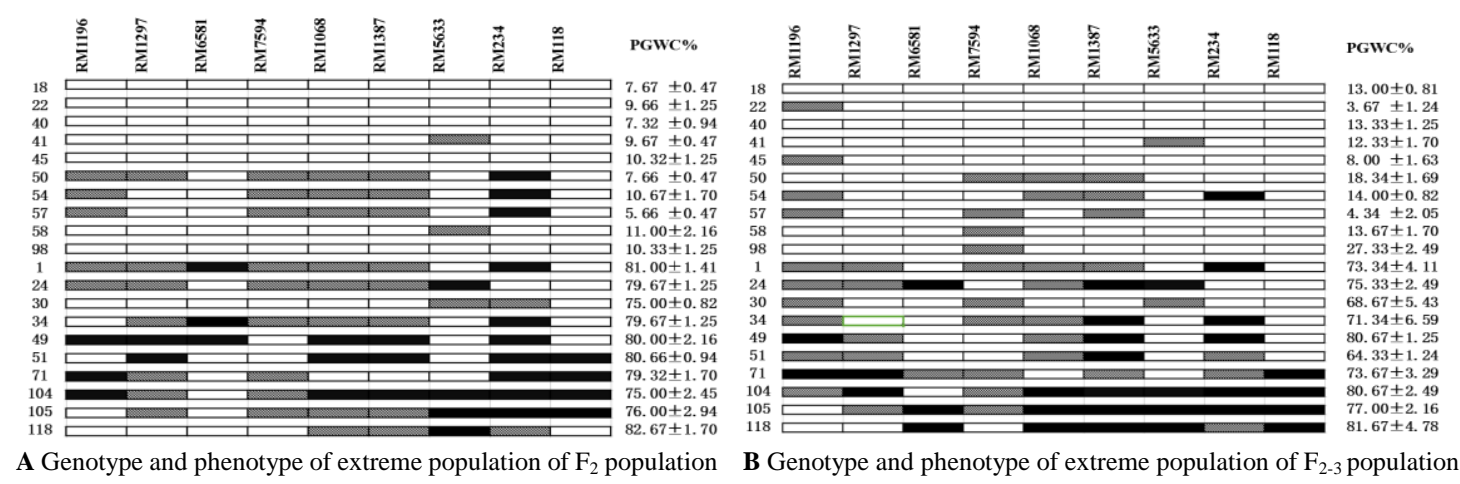

Fig 1. Genotypes and phenotypes of extreme individual plant of $F_{2}$ and $F_{2: 3}$ family lines. The white, black and diagonal bars denote the genotype of Huanghuazhan, CS 197 and heterozygote respectively.

\section{Genetic Separation of Phenotypes of PGWC}

The PGWC of CS197 and Huanghuazhan were $81.67 \%$ and $10.33 \%$, respectively. There were $5.66-82.67 \%$ of the chalky grain size distribution in the $\mathrm{F}_{2}$ population, $31.67 \%$ in the largest range of PGWC, and $3.33 \%$ in the smallest interval of $80-90 \%$, with an average value of $35.27 \%$ lower than the $F_{1}$ average of $39.33 \%$, the coefficient of variation was $57.13 \%$. The broad sense heritability was $26.31 \%$, while the narrow heritability sense was $17.21 \%$. The environmental variance was $42.10 \%$, and the average dominant degree was 0.78 . The super-parent strain genotype Huanghuazhan accounted for $75 \%$, that of CS197 was $11.11 \%$ and that of heterozygosity was $13.89 \%$. The quality traits of 10 extremely low PGWC were similar to those of parental Huanghuazhan, while those of 10 extremely high PGWC were similar to those of parental CS197 (Table 1). 
Table1 . Quality traits of extreme single plant in $\mathrm{F}_{2}$ populations of Huanghuazhan / CS197. ACE, area of chalky endosperm ; BR, Brown rice percentage; HR, Head rice percentage ; AC, Amylose content ;

GL ,Grain length ; GW, Grain width ; LWR, length-width ratio ; GS, grain shape.

\begin{tabular}{|c|c|c|c|c|c|c|c|c|c|c|c|c|}
\hline Traits & Material & PGWC/\% & ACE $/ \%$ & $\mathrm{DEC} / \%$ & $\mathrm{BR} / \%$ & $\mathrm{MR} / \%$ & $\mathrm{HR} / \%$ & $\mathrm{AC} / \%$ & $\mathrm{GL} / \mathrm{mm}$ & $\mathrm{GW} / \mathrm{mm}$ & LWR & GS \\
\hline \multirow{2}{*}{ Parents } & Huanghuazhan & $10.32 \pm 2.33$ & $2.31 \pm 1.24$ & $2.45 \pm 0.47$ & $77.17 \pm 1.01$ & $68.49 \pm 0.59$ & $58.87 \pm 4.64$ & $15.18+0.01$ & $6.66 \pm 0.06$ & $1.86 \pm 0.03$ & $3.57 \pm 0.08$ & length \\
\hline & CS197 & $81.67 \pm 10.35$ & $82.67 \pm 0.94$ & $65.51 \pm 1.70$ & $82.53 \pm 2.13$ & $71.34 \pm 0.72$ & $61.63 \pm 2.59$ & $7.33 \pm 0.04$ & $5.76 \pm 0.08$ & $2.79 \pm 0.07$ & $2.06 \pm 0.04$ & Oval \\
\hline \multirow{10}{*}{$\begin{array}{l}\text { Low } \\
\text { PGWC } \\
\text { lines }\end{array}$} & 18 & $7.67 \pm 0.47$ & $15.66 \pm 1.70$ & $11.11 \pm 1.24$ & $77.36 \pm 1.25$ & $67.97 \pm 1.69$ & $56.36 \pm 1.95$ & $14.26 \pm 0.03$ & $6.78 \pm 0.11$ & $1.80 \pm 0.06$ & $3.76 \pm 0.14$ & length \\
\hline & 22 & $9.66 \pm 1.25$ & $26.67 \pm 0.47$ & $26.76 \pm 1.69$ & $78.00 \pm 1.60$ & $68.53 \pm 0.64$ & $59.60 \pm 1.26$ & $10.26 \pm 0.11$ & $6.54 \pm 0.23$ & $1.76 \pm 0.06$ & $3.41 \pm 0.03$ & length \\
\hline & 40 & $7.32 \pm 0.94$ & $6.33 \pm 1.25$ & $5.63 \pm 1.24$ & $75.24 \pm 1.20$ & $69.00 \pm 0.93$ & $58.36 \pm 1.79$ & $14.60 \pm 0.08$ & $6.62 \pm 0.19$ & $1.94 \pm 0.07$ & $3.06 \pm 0.08$ & length \\
\hline & 41 & $9.67 \pm 0.47$ & $11.66 \pm 1.24$ & $10.28+1.70$ & $74.31 \pm 2.77$ & $68.21 \pm 1.75$ & $57.47 \pm 0.97$ & $12.72 \pm 0.05$ & $6.65 \pm 0.26$ & $2.17 \pm 0.11$ & $3.31 \pm 0.09$ & length \\
\hline & 45 & $10.32 \pm 1.25$ & $8.67 \pm 1.25$ & $7.95 \pm 0.94$ & $75.78+1.20$ & $69.32 \pm 0.73$ & $57.70 \pm 0.50$ & $12.42 \pm 0.04$ & $6.73 \pm 0.18$ & $2.03 \pm 0.10$ & $3.29 \pm 0.14$ & length \\
\hline & 50 & $7.66 \pm 0.47$ & $5.33 \pm 2.05$ & $4.08 \pm 1.63$ & $80.70 \pm 2.39$ & $66.73 \pm 0.58$ & $58.21 \pm 0.86$ & $14.58+0.05$ & $6.57 \pm 0.20$ & $2.00 \pm 0.11$ & $3.34 \pm 0.16$ & length \\
\hline & 54 & $10.67 \pm 1.70$ & $5.64 \pm 1.25$ & $6.72 \pm 2.16$ & $76.72 \pm 2.87$ & $68.76 \pm 1.13$ & $59.06 \pm 0.93$ & $14.49 \pm 0.04$ & $6.48 \pm 0.08$ & $1.95 \pm 0.08$ & $3.52 \pm 0.24$ & length \\
\hline & 57 & $5.66 \pm 0.47$ & $8.66 \pm 1.70$ & $5.31 \pm 1.69$ & $75.98 \pm 2.86$ & $68.27 \pm 0.95$ & $58.08 \pm 0.86$ & $15.50 \pm 0.05$ & $6.74 \pm 0.30$ & $1.98 \pm 0.17$ & $3.42 \pm 0.15$ & length \\
\hline & 58 & $11.00 \pm 2.16$ & $6.32 \pm 1.25$ & $7.52 \pm 1.88$ & $80.19 \pm 1.61$ & $70.06 \pm 0.90$ & $56.78 \pm 2.63$ & $14.98 \pm 0.03$ & $6.23 \pm 0.05$ & $1.77 \pm 0.12$ & $3.52 \pm 0.24$ & length \\
\hline & 98 & $10.33 \pm 1.25$ & $12.67 \pm 1.24$ & $14.09 \pm 0.47$ & $76.41 \pm 0.43$ & $69.59 \pm 0.49$ & $58.25 \pm 1.10$ & $14.09 \pm 0.07$ & $6.45 \pm 0.16$ & $1.94 \pm 0.15$ & $3.35 \pm 0.29$ & length \\
\hline \multicolumn{2}{|c|}{ Range } & $5.66-11.00$ & $5.33-26.67$ & $4.88-25.76$ & $74.31-80.70$ & $66.73-69.59$ & $56.36-59.60$ & $10.26-15.50$ & $6.23-6.78$ & $1.77-2.17$ & $3.06-3.76$ & \\
\hline \multicolumn{2}{|c|}{ Average } & 9.00 & 10.76 & 9.94 & 77.11 & 68.65 & 57.99 & 13.79 & 6.58 & 1.93 & 3.42 & \\
\hline \multicolumn{2}{|c|}{$\mathrm{CV} / \%$} & 18.81 & 57.69 & 63.10 & 2.56 & 1.29 & 1.58 & 16.90 & 4.08 & 13.55 & 12.25 & \\
\hline \multirow{10}{*}{$\begin{array}{l}\text { High } \\
\text { PGWC } \\
\text { lines }\end{array}$} & 1 & $81.00 \pm 1.41$ & $92.67 \pm 8.99$ & $78.32 \pm 1.24$ & $81.91 \pm 1.12$ & $71.23 \pm 0.48$ & $63.05 \pm 0.48$ & $7.27 \pm 0.03$ & $5.44 \pm 0.16$ & $2.85 \pm 0.04$ & $1.92 \pm 0.07$ & Oval \\
\hline & 24 & $79.67 \pm 1.25$ & $31.33 \pm 1.25$ & $23.66 \pm 0.47$ & $81.79 \pm 1.53$ & $71.32 \pm 1.14$ & $61.96 \pm 0.47$ & $5.18 \pm 0.02$ & $5.76 \pm 0.04$ & $2.87 \pm 0.07$ & $2.01 \pm 0.05$ & Oval \\
\hline & 30 & $75.00 \pm 0.82$ & $36.00 \pm 0.82$ & $26.67 \pm 1.24$ & $80.75 \pm 0.63$ & $70.05 \pm 0.93$ & $63.47 \pm 0.79$ & $9.95 \pm 0.04$ & $5.90 \pm 0.14$ & $2.75 \pm 0.06$ & $2.14 \pm 0.08$ & Oval \\
\hline & 34 & $79.67 \pm 1.25$ & $56.34 \pm 1.69$ & $47.00 \pm 2.16$ & $81.48 \pm 0.75$ & $72.61 \pm 0.46$ & $62.73 \pm 0.77$ & $14.10 \pm 0.02$ & $5.84 \pm 0.16$ & $2.83 \pm 0.08$ & $2.06 \pm 0.01$ & Oval \\
\hline & 49 & $80.00 \pm 2.16$ & $25.00 \pm 2.16$ & $18.67 \pm 1.69$ & $82.09 \pm 0.19$ & $72.51 \pm 0.58$ & $62.46 \pm 0.85$ & $7.74 \pm 0.03$ & $5.95 \pm 0.11$ & $2.71 \pm 0.07$ & $2.20 \pm 0.10$ & Oval \\
\hline & 51 & $80.66 \pm 0.94$ & $56.66 \pm 4.03$ & $44.33 \pm 3.09$ & $82.61 \pm 0.44$ & $71.69 \pm 1.13$ & $62.21 \pm 0.75$ & $9.27 \pm 0.04$ & $6.04 \pm 0.21$ & $3.01 \pm 0.05$ & $2.00 \pm 0.06$ & Oval \\
\hline & 71 & $79.32 \pm 1.70$ & $72.67 \pm 3.29$ & $59.67 \pm 2.05$ & $82.12 \pm 1.12$ & $72.77 \pm 0.36$ & $62.71 \pm 1.16$ & $8.34 \pm 0.02$ & $6.43 \pm 0.54$ & $2.71 \pm 0.05$ & $2.37 \pm 0.24$ & Oval \\
\hline & 104 & $75.00 \pm 2.45$ & $52.32 \pm 2.49$ & $40.00 \pm 6.53$ & $81.56 \pm 0.89$ & $71.45 \pm 1.48$ & $61.99 \pm 0.34$ & $11.59 \pm 0.02$ & $6.47 \pm 0.69$ & $2.86 \pm 0.04$ & $2.26 \pm 0.25$ & Oval \\
\hline & 105 & $76.00 \pm 2.94$ & $66.00 \pm 7.79$ & $46.34 \pm 2.05$ & $80.89 \pm 1.31$ & $72.34 \pm 1.51$ & $62.00 \pm 2.43$ & $9.74 \pm 0.03$ & $6.33 \pm 0.46$ & $2.64 \pm 0.12$ & $2.40 \pm 0.25$ & Oval \\
\hline & 118 & $82.67 \pm 1.70$ & $73.67 \pm 3.30$ & $62.66 \pm 2.05$ & $81.84 \pm 1.11$ & $72.55 \pm 0.57$ & $62.32 \pm 0.72$ & $10.91 \pm 0.04$ & $6.43 \pm 0.74$ & $2.81 \pm 0.20$ & $2.28 \pm 0.11$ & Oval \\
\hline \multicolumn{2}{|c|}{ Range } & $75.00-82.67$ & $31.33-92.67$ & $18.67-78.32$ & $80.75-82.61$ & $70.05-72.77$ & $61.96-63.47$ & $5.18-14.10$ & $5.44-6.47$ & 2.643 .01 & $1.92-2.40$ & \\
\hline \multicolumn{2}{|c|}{ Average } & 78.90 & 56.27 & 44.73 & 81.71 & 71.85 & 62.49 & 9.41 & 6.06 & 2.81 & 2.17 & \\
\hline \multicolumn{2}{|c|}{ CV/\% } & 3.18 & 35.62 & 39.69 & 0.65 & 1.34 & 0.76 & 25.11 & 5.38 & 3.54 & 7.16 & \\
\hline
\end{tabular}

\section{Discussion}

The PGWC is one of the main appearance qualities of rice. Huanghuazhan in china has been approved by Guangdong, Hunan, Hubei, Guangxi, Hainan, Jiangxi, Zhejiang, Chongqing and Shanxi provinces. It is the first rice varieties of grade I based on national standard promoted in large rice area of southern China. PGWC of Huanghuazhan was very low, and the results from Guangdong and Hunan were 4\%. Prepareing the hybrid combinations based on Huanghuazhan to study the phenotypic and genotypic conformational mechanisms of PGWC in $F_{2}$ isolates is important to improve the selection efficiency of chrysanthemum grain yield in hybrid offspring, and to utilize Huanghuazhan and hybrid rice quality breeding.

With the development of genomics and molecular marker technology, the researchers used QTLs to locate PGWC genes in various populations, which were proved be distributed on 12 chromosomes in rice [2-3,7-9]. Liu et al. [12] detected three major QTLs affecting the PGWC on the 5th, 8th and 10th chromosomes using the RIL population. Tan et al. [8] used $\mathrm{F}_{2}$ population to detect QTLs associated with PGWC on chromosomes 1, 3, 5, 6 and 7. In this study, Using the molecular marker detection and phenotypic identification in the $\mathrm{F}_{2}$ population of extreme individuals and the corresponding $\mathrm{F}_{2: 3}$ families, the results found the two generations of similar results, with a high degree of compliance. It is shown that the selection efficiency of chalky grain rate can be improved by double selection of phenotype and molecular marker.

In the practice of hybrid rice breeding, the greater the separation of the $\mathrm{F}_{2}$ generation, the greater chance of selecting the target strain. Breeding selection is difficult, especially the low trait. In this study, the chalkiness rate of hybrid $\mathrm{F}_{2}$ population was 5.66-82.67\%, and was continuous distribution. The maximum value was close to $81.67 \%$ of CS197 and the lowest value is lower than the PGWC of parents Huanghuazhan, accounted for $10.32 \%$. PGWC distribution of the largest interval of 
$20-30 \%$ accounted for $31.67 \%$ and the smallest interval of $80-90 \%$ of the strains accounted for $3.33 \%$. PGWC distribution trend to female parent Huanghuazhan with low PGWC. Chio [13] also found that the chalkiness of the hybrid was influenced by the maternal effect and showed a sense of inheritance.

Reducing chalky grain rate is important for improving rice quality. Although the PGWC is affected by genetic and environmental effects, it is important to explore the genetic mechanism of PGWC genes in its offspring by studying the genetic separation of PGWC, which can play an important role in hybrid rice quality breeding. In this study, the PGWC of the individual isolates of the $F_{2}$ isolates was analyzed. The phenotypic characterization of the low PGWC and high $\mathrm{PGWC} \mathrm{F}_{2}$ and $\mathrm{F}_{2: 3}$ families was observed by Statistical Analysis. The phenotypic and genotypic mechanisms were studied, and the low PGWC lines were used as hybrid parents. The application of phenotype and genotype to the prediction of heterosis, effective selection of low PGWC lines will reduce the blindness selection, and has an important role in high quality rice breeding

\section{Conclusion}

The results revealed that the genotypesand and phenotype of 10 extremely low PGWC individual plants were the similar to Huanghuazhan. Phenotype of the most 10 extremely high individual plants were similar to CS197.

\section{Acknowledgments}

This research was supported by the key Natural Science Foundation of Chongqing (CSTC2015jcyjBX0091), National Natural Sciences Foundation (31670326), The Program for Innovative Research Team in University, Chongqing (CXTDX201601018).

\section{References}

[1] FAO. FAO statistical yearbook 2013 (2013-12-01) www.fao.org / docrep/ 018/ i3107e / i3107e00. Htm

[2] Bian J M, Shi H, Li C J, Zhu C L, Yu Q Y, Peng X S, Fu J R, He X P, Chen X R, Hu LF, Ouyang L J, He H H. QTL mapping and correlation analysis for 1000-grain weight and percentage of grains with chalkiness in rice. Journal of Genetics, 2013, 2(9): 281-287.

[3] Zhou L J, Chen L M, Jiang L, Zhang W W, Liu L L, Liu X, Zhao Z G, Liu S J, Zhang L J, Wang J K, Wan J M. Fine mapping of the grain chalkiness QTL qPGWC-7 in rice (Oryza sativa L.). Theor Appl Genet, 2009, 118: 581-590.

[4] Kou Y C, Liu C. Inheritance of chalkiness of rice endosperm. Agric Res China, 1986, 35(2): 129-138.

[5] Huang H Q, Zhou X Y, Analysis of Combining Ability of Rice Chalkiness. Hunan Agricaltural Sciences, 1991, 9(1): 18-20. (In Chinese)

[6] Edwards K, John Stone C, Thompson C, A simple and rapid method for the preparation of plant genomic DNA for PCR Analysis. Nucl Acids Res, 1991, 19: 1349. 
[7] Guo T, Liu X L, Wan X Y, Weng J F, Liu S J, Liu X, Chen M J, Li J J, Su N, Wu F Q, Cheng Z J, Guo X P, Lei C L, Wang J L, Jiang L, Wan X Y. Identification of a Stable Quantitative Trait Locus for Percentage Grains with White Chalkiness in Rice (Oryza sativa). Journal of Intergrative Plant Biology, 2011, 53(8): 598-607.

[8] Tan Y F, Xing Y Z, Li J X, Yu S B, Xu C G, Zhang Q F. Genetic bases of appearance quality of rice grain in Shanyou 63.an elite rice hybrid. Theor Appl Genet, 2000, 101: 823-829.

[9] Peng B, Wang L Q, Fan C C, Jiang G H, Luo L J, Li Y B, He Y Q. Comparative mapping of chalkiness components in rice using five populations across two environments. BMC Genetics, 2014, 15(1): 49-63.

[10] Li Z F, Wan J F, Xia J F, Zhai H Q. Mapping quantitative trait loci undering appearance quality of rice grains (Oryza sativa L.). Acta Genet Sin, 2003, 30(3): 251-259.

[11] Zhou L J, Jiang L, Liu X, Chen H, Chen L M, Liu S J, Wan J M. QTL mapping and interaction analysis for 1000-grain weight and percentage of grains with chalkiness in rice. Acta Agron Sin, 2009, 35: 255-261. (In Chinese)

[12] Liu X, Wang Y, Wang S W. QTL analysis of percentage of grainswith chalkiness in japonica rice ( Oryza sativa). Genet MolRes, 2012, 11( 1): 717-724.

[13] Chol S. J. Seoul National University. College of Agriculture Bullttin. 1979, 4(1): 247-276. 